\title{
What Kind of Kind Is Intelligence?
}

\author{
Davide Serpico
}

Department of Classics, Philosophy and History, University of Genoa, Italy

\begin{abstract}
The model of human intelligence that is most widely adopted derives from psychometrics and behavioral genetics. This standard approach conceives intelligence as a general cognitive ability that is genetically highly heritable and describable using quantitative traits analysis. The paper analyzes intelligence within the debate on natural kinds and contends that the general intelligence conceptualization does not carve psychological nature at its joints. Moreover, I argue that this model assumes an essentialist perspective. As an alternative, I consider an HPC theory of intelligence and evaluate how it deals with essentialism and with intuitions coming from cognitive science. Finally, I highlight some concerns about the HPC model as well, and conclude by suggesting that it is unnecessary to treat intelligence as a kind in any sense.
\end{abstract}

Keywords: Intelligence; IQ; $\mathrm{g}$ Factor; Natural Kinds; Homeostatic Property Cluster; Essentialism; Psychometrics; Behavioral Genetics; Heritability

\section{Introduction}

Although the concept of intelligence is shrouded in controversy, it is nonetheless widely used both in psychological and in folk settings. Several theories have been proposed in order to clarify whether intelligence can be conceived as a general ability or only as a bundle of distinct cognitive phenomena. Several criticisms have been raised against a strong interpretation of genetic data based on the well-known intelligence quotient (IQ). Several attempts have been made to find more comprehensive definitions of intelligence. After a century of research, there is still extensive debate going on about the status of intelligence.

Almost any actively involved scholar recognizes the social consequences of their research. These sometimes can deeply affect social fields stretching from politics to education and psychiatry. While questions about intelligence have typically involved empirical problems related to genetics or psychology, I shall offer a conceptual analysis of the subject matter, involving natural kinds theory. I focus in particular on what Douglas Wahlsten (2002) calls "the theory of biological intelligence", a model often quoted in psychometrics and behavioral genetics. This model is rooted in research over the last century. It is not the only one available, but the majority of alternative theories of intelligence have been proposed to deal with issues deriving from that original model.

In $\S 1$, I introduce the standard model of intelligence and describe the scientific controversies around it. In this section, I also introduce natural kinds theory, explaining 
why it represents a useful tool for arriving at a better understanding of intelligence. In $\S 2$ and $\S 3$, I clarify the essentialist assumptions underlying intelligence research, appealing to heritability and molecular research. In $\S 4$, I propose a reconstruction of psychometric intelligence as a homeostatic property cluster (HPC) in order to avoid essentialism and to account for some common intuitions about cognition. Finally, in $\S 5$, I put my own proposal that it is unnecessary to conceive intelligence as a unified cognitive phenomenon or as a kind.

\section{The Theory of Biological Intelligence}

Over the last century, intelligence studies have been developed mainly within two scientific fields: psychometrics and behavioral genetics. Although driven by different research purposes, both have provided a quantitative analysis of intelligence.

Psychometrics studies intelligence in light of two theoretical constructs: IQ and $g$ factor. Since IQ and $g$ are strongly correlated to one another, the relative terms are sometimes treated as synonymous. Nonetheless, they should be kept conceptually distinct. Given a particular population, the IQ level changes among individuals according to a bell curve. Thus, IQ stands for the individual intelligence level assessed by tests. In contrast, $g$. stands for two different things: on the one hand, it derives from a factor analysis as the outcome of correlation matrices of cognitive test scores; on the other hand, it is, broadly speaking, a construct offered to explain IQ. In this second sense, $g$ is conceived as a general cognitive ability that underlies individual test performances. According to this approach, whatever intelligence is, it is measurable by IQ tests, while $g$ explains individual intellectual differences.

Behavioral genetics, in turn, investigates intelligence using methods derived from the genetics of quantitative traits in order to understand the role of inheritance in accounting for individual differences. Like other quantitative traits, such as height and weight, intelligence occurs as a continuous range of variation within populations. In order to account for these traits, geneticists adopted the additive polygenic model of inheritance: accordingly, several genes act additively on the phenotype-in this case, a person's intellectual level. For many decades, the main goal of behavioral genetics has been to understand how relevant inheritance is in explaining individual differences and similarities and has the psychometric measure IQ been adopted as a good 'marker' of individual intelligence.

Over time, the purposes of both areas have converged, resulting in a model which is still influential: the theory of biological intelligence. After the adoption of the $g$ factor by genetic research, a consensus has been reached around a model of intelligence as a highly heritable general cognitive ability. Despite widespread disagreement about $g^{2} \mathrm{~s}$ psychobiological meaning, the psychometric-genetic approach (henceforth PSY-GEN) takes $g$ as a prominent psychological variable (Jensen, 2002): " $g$ is one of the most 
reliable and valid measures in the behavioral domain" (Plomin et al., 2013, p. 187). However, neither intelligence nor the $g$ factor has figured prominently in the cognitive sciences. Since IQ test skills clearly belong to the cognitive domain, this might strike one as surprising. The attempt to bridge psychometrics and cognitive science is relatively recent-especially considering that the psychometric approach to intelligence dates back to the early twentieth century (see Pretz \& Sternberg, 2005).

\subsection{Ontological issues}

The existence of a general factor of intelligence was first proposed by Charles Spearman (1904). His reasons were straightforward: intelligence measurements are positively related to one other: if one shows good performance on a given task, one tends to show good performance on other tasks (to a varying degree). This is called the 'positive manifold'. Thus, $g$ is a summary index of a correlation matrix, representing what cognitive tests have in common and explaining $\sim 40 \%$ of their variance (Plomin et al., 2013 , p. 210). In this respect, it is fairly uncontroversial. ${ }^{1}$

The subject of controversy lies in the psychobiological nature of $g$ : PSY-GEN does not interpret $g$ in any strong ontological sense. As is often the case in psychometrics, a clearcut distinction between methodological purposes and the reality of a psychological construct is endorsed. In other words, psychometrics does not investigate what $g$ is; it is sufficient to ensure that IQ tests are capable of evaluating intelligence-whatever it is. Nonetheless, in light of the positive manifold, several psychologists have posited the existence of an underlying general mental ability (Van der Maas et al., 2006). Hence, some ontologically driven hypotheses were put forward, especially when psychometrics came face to face with the biological sciences. The advocates of $g$ conceive this factor as a cognitive entity responsible for individual differences in test performances. The degree of the realist commitment is different depending on the authors' standpoint, but most of them admit $g$ as a psychobiological characteristic which influences intelligent behavior. ${ }^{2}$ For instance, Spearman described $g$ as a form of mental energy. More recently, in order to guarantee that $g$ is a valid measure of intelligence, some scholars have tried to relate it to constructs from cognitive neuroscience-e.g., working memory, processing speed, neural efficiency and brain size (Pretz \& Sternberg, 2005). Briefly, this research assumes $g$ must exist somehow: we can look for its biological correlates in order to ground it in other cognitive phenomena which seem to exist and are not posited merely on the basis

\footnotetext{
${ }^{1}$ Gould (1981), among others, criticized this point by referring to particular interpretations of factor analysis. Indeed, this statistical method could lead to a $g$ factor or not, depending on technical choices. Since most scholars hold that $g$ is a well-established psychometric entity, I do not take into consideration these criticisms.

${ }^{2}$ For the sake of a case of disagreement, Humphreys and Stark (2002), although they are psychometricians, do not accept $g$ as a fixed biological capacity and argue against reification. The reification of $g$ is stronger in those authors who emphasize the role of biological sciences (for loose and strict interpretations of $g$, see Kray and Frensch, 2002,).
} 
of statistical research.

By contrast, several authors cast doubt on such strong interpretations of $g$. According to Kray and Frensch (2002), there is no convincing empirical evidence that supports the existence of $g$. For Stankov (2002, p. 35), "there is no single cognitive process that can explain the presence of $g[\ldots]$ It is a mixture of many different processes (including noncognitive influences) that are known to change in the course of development" (see also Naglieri \& Das, 2002).

Although the advocates of the PSY-GEN approach rarely engage in philosophical debates, it is fruitful to discuss the theory of biological intelligence and natural kinds theory conjointly. Several efforts have been made to establish whether psychological constructs are natural kinds. ${ }^{3}$ These efforts are aimed at exploring the extent and degree to which such concepts meet ontological and epistemological requirements imposed by natural kinds theory. Within this theoretical framework, a link is assumed between scientific realism and natural kinds. ${ }^{4}$ Introducing natural kinds allows us to offer a framework within which we can evaluate the ontological status of psychological constructs apart from our scientific theories and categorizations (i.e., as mind-independent). Proponents of the realist view on natural kinds assume that science is able "to carve nature at its joints": entities identified and classified by science correspond to real kinds in nature and trace natural properties and relations. For instance, discovering biological pathologies correlated to mental disorders (lesions and genetic issues) is often considered analogous to discovering the atomic number of a chemical element (Zachar, 2014). ${ }^{5}$

If PSY-GEN was brought into the philosophical debate, it would be regarded as a prototypical theory: thinking of the notion of intelligence as a natural kind would be a way (likely, not the only one) to say that intelligence deserves a place in our ontology, or a way to exert a clear ontological commitment about general intelligence or about $g$. I will explore this hypothesis. If intelligence were a kind, one may ask what kind of kind it would be. The most promising hypothesis points to the HPC theory, introduced by Richard Boyd (1991), for three main reasons: the first concerns limitations of traditional essentialism; the second depends on the fact that Boyd's theory seems suitable for tackling anti-realism; the last pertains to multilevel analysis. I extensively discuss these points in $\S 3.2$.

Can the PSY-GEN model of intelligence be accounted for from a realist perspective? If the answer were positive, then this model would reflect the way in which cognition works on a biological level-briefly, it would carve psychological nature at its joints. In particular, term " $g$ " would denote something in the world that should be admitted along with

\footnotetext{
${ }^{3}$ Renowned examples are emotions (Barrett, 2006) and psychiatric disorders (Kincaid \& Sullivan, Eds., 2014).

${ }^{4}$ For a discussion about realism and natural kinds, see Franklin-Hall (2015).

${ }^{5}$ Natural kinds have been invoked in semantic, metaphysic and scientific debates. It is not my aim to evaluate which is the best way to understand them; rather, I mainly assume a metaphysical standpointalthough I do not exclude some epistemological concerns.
} 
other neurocognitive processes or brain architectures into the realm of trustworthy concepts.

\subsection{How does Behavioral Genetics Conceptualize General Intelligence? ${ }^{6}$}

Since Francis Galton' studies in the nineteenth century, intelligence has been considered a quantitative trait-something shared by all human beings but expressed differently among them. Like height and weight, intelligence manifests a continuous range of variation. Such an intuition has led to the quantification of intellectual manifestations and the categorization of people. Although practical applications have changed, largely depending on social circumstances, the chief aim of testing is to measure, while IQ stands for that number which is useful to sort individuals according to their intellectual features.

Having a technical tool for categorization has applications across several social areas, from education to job recruiting. Here I consider mainly the clinical impact, concerning intellectual disability. The most recent versions of DSM (Diagnostic and Statistical Manual of Mental Disorders) categorize intellectual disability as a clinical condition characterized by various diagnostic criteria, among which the first pertains to low IQ level. Other criteria address adaptive functioning for social standards and intellectual and adaptive deficits during development. Behavioral genetics also invokes IQ, focusing on so-called "general cognitive disability", which concerns only low IQ (Plomin et al., 2013, p. 163). Four degrees of severity are generally distinguished: profound (IQ $<20$ ), severe (20 to 35$)$, moderate (35 to 50 ) and mild (50 to 70$).^{7}$

Behavioral genetic research supports high heritability indexes, up to 80\% (see Bouchard, 2004, for a review). Low IQ seems as heritable as IQ in the normal range (Plomin et al., 2013, p. 165). According to behavioral geneticists, it is indubitable that inheritance plays a key role in intellectual development. What about the relationship between genes and intelligence? Since intelligence is a quantitative trait, it has generally been assumed that different genes make additive contributions to IQ level-this is known as the additive polygenic model of inheritance (Mather, 1943). Proponents of this model to not seek a specific gene for intelligence; rather, many genes are assumed to be involved in the phenomenon and no single gene, taken in isolation, determines intelligence. According to this model, both genes and environment contribute additively to the phenotype and there is no significant interaction between them.

Qualitative traits, like the ones analyzed in Mendelian genetics, allow categorical

\footnotetext{
${ }^{6}$ I mainly refer to Plomin et al. (2013), summarizing a synthesis of the genetic approach to human behavior.

${ }^{7}$ DSM-V changed severity criteria, that now concern the general adaptive functioning of the individual (American Psychiatric Association, 2013, p. 33). However, Plomin et al. (2013, p. 163) refer to DSM-IVTR criteria.
} 
reasoning. This is not the case for quantitative traits. Thus, the quantitative conception of intelligence seems incompatible with natural kinds theory. Indeed, it is not immediately clear what the role of quantitative traits in a kinds framework would be. Nick Haslam (2014) states that natural kinds involve categories rather than dimensionstraits distributed on a dimension are not kinds in the narrow sense. Haslam's intuition is convincing. ${ }^{8}$ Often, when one speaks about kinds, one has in mind a set of properties. Classically, natural kinds have been involved in the relationship between properties and classes (see Hacking, 1991). Of course, this is not the only issue related to natural kinds. However, focusing on properties became prominent in many theoretical contexts after Boyd's HPC theory was adopted by many authors across several fields, from psychology to natural sciences.

Natural kinds theories have assumed the ontological status of these properties, and explored how they combine and whether they are necessary and/or sufficient to define a kind. Let us consider, for example, psychiatry: when we ask whether or not a mental disorder should be considered as a natural kind, we pick up a collection of properties (behaviors and symptoms) and analyze the relationship between these properties to determine whether one of them is necessary, for an individual, to be an instance of the kind.

Here the situation appears to be different. First, intelligence is a dimensional phenomenon. As measured by intelligence tests it situates individuals along a bell curve. However, intelligence is not solely something dimensional because of this statistical feature: it is hypothesized to be a quantitative trait in itself. As I said above, the way in which genes influence intelligence is quantitative. Secondly, from a genetics point of view, $g$ is not a property cluster-that is, a collection of properties in a Boydian sense. Rather, it is a single property, a unified psychological trait shared by every human being in varying degrees. For instance, this variable could figure among other symptoms in diagnosing a mental disorder: in fact, intellectual disability is a medical condition in which low IQ figures as a symptom, or a diagnostic criterion, among others. Generally, the proper targets of a natural kinds inquiry are not the symptoms (the properties), but rather the disorder itself (the cluster). For instance, low mood could be intended as something dimensional, too. However, it does not make sense to ask whether it is a natural kind: at most, it would be a necessary (but not sufficient) property to define mood disorders such as major depression.

Although I am arguing for a kind view of intelligence, it is important to notice that it is not trivial for geneticists to accept this analysis. It may seem similar to height, just another quantitative trait which would represent a single property unlikely to be useful for the natural kinds theory. ${ }^{9}$ What would the property cluster be? What are the

\footnotetext{
${ }^{8}$ John Dupré (personal communication, April, 2016) cast similar doubts: could it be possible to account for variables in a natural kinds theory? To fully address this question would go beyond my purposes. I shall show that we are in need of an argument to arrange general intelligence within a kind framework.

${ }^{9}$ Quantitative analyses of height are quite similar to those applied to IQ (see Visscher, 2008).
} 
membership criteria for such a kind? I shall now show that this theory of intelligence, related to quantitative traits analysis, implies a particular type of essentialism, which Paul Griffiths (2002) referred to as "folk essentialism".

\section{A Folk-Essentialist Tendency}

For a few decades, essentialism has been linked to natural kinds. In our case, we are not dealing with the so-called 'traditional essentialism', which often involves discourses about kinds and properties. Rather, we focus on a tacit essentialist assumption that underlies several scientific debates. Folk essentialism is "a distinctive feature of prescientific thought about animate things. [It] understands biological species as the manifestation of underlying 'natures' shared by all members of a species" (Griffiths, 2002, p. 72). Like traditional essentialism, folk essentialism explains similarities by referring to underlying shared properties. However, folk essentialism does not appeal to any theoretical understanding: it simply picks out a psychological tendency.

Folk essentialism underlies controversies about innateness. Indeed, Griffiths described the innateness concept as an expression of folk essentialism. It posits that the development of an innate trait is established before birth and is inflexible. Behavioral differences are thus grounded in genetic differences (see also Samuels, 2004). Behavioral geneticists frequently seem to think in terms of folk essentialism. In this respect, I will consider heritability analysis, a popular statistical methodology introduced by population genetics in order to analyze how populations react to selection.

\subsection{The heritability controversy}

When applied to human traits, heritability analysis generates strong disagreement, mostly concerning what heritability could tell us and what it was often "pushed" to tell. Heritability is the proportion (measured between 0 and 1) of phenotypic variance that is attributable to genotypic variance. In other words, a heritability index evaluates to what extent variation in a phenotypic trait-within a population, in a given environmentcould be explained by genetic variation among individuals. If heritability is high, the variability is probably due to genetic differences among individuals. If it is low, the variability is probably attributable to environment.

As has already been mentioned, geneticists estimated high heritability for IQ-generally between 50\% and 80\%. Detractors of heritability research (e.g., Block, 1995; Joseph, 2004) state that from these data alone one cannot legitimately infer anything about genetic influence on a phenotypic trait. The concepts 'innate', 'genetic' and 'heritable' have no clear connection with each other. To say that genetic variation explains $80 \%$ of variance of a trait in a population does not mean that this trait in an individual is $80 \%$ 
influenced by genes and $20 \%$ by environment; nor it does mean that an individual has an $80 \%$ chance of inheriting it. ${ }^{10}$

Nonetheless, jumping from heritability to determinism is very frequent. It is manifest in very popular simplifications ("this behavior is innate", "it is part of human nature", "the gene for...has been found", etc.). The fact that heritability is expressed as a percentage has led some scholars to the conclusion that it gives us a quantitative appraisal of the relevance of genes in determining a phenotypic trait. The term often used is the magnitude of inheritance-and, by subtraction, of environment-for individual differences (e.g., Bouchard, 2004). Thus, high heritability of IQ seems to imply that inheritance must play a key role in intelligent behavior. For instance, Chabris et al. (2012, pp. 1-2) link heritability and inheritance: "if a trait is heritable in the general population, then in principle it should be possible [...] to identify molecular genetic variants that are associated with the trait. General cognitive ability, or $g,[\ldots]$ is one of the most heritable behavioral traits. Estimates of broad heritability as high as 0.80 have been reported for adult IQ in modern Western populations". According to detractors of heritability research, this is by definition wrong. As I will show, the improper use of heritability data relies on folk-essentialist assumptions.

\subsection{Essentialism via heritability research}

Folk essentialism is strictly related to the idea that the development of an innate trait is established in advance or, at least, is hard to change and insensible to external influences. Such a perspective is pervasive in psychometric-genetic literature. Generally speaking, the assumption that an IQ test could tell us something about the intellectual destiny of anyone has inspired public policies and their ethical and societal consequences. For instance, developmental fixity has led to implementation of educational policies that direct children towards a particular educational path according to their intellectual aptitudes. This is by no means something new. In a classical publication, Arthur Jensen stated: "currently used IQ tests do indeed reflect innate, genetically determined aspects of intellectual ability in persons from the population on which the tests were standardized and validated" (Jensen, 1969, p. 8).

Consider data from a popular longitudinal project called MISTRA (Minnesota Study of Twins Reared Apart, Bouchard et al., 1990). The team, headed by Thomas Bouchard, analyzed many different traits of MZ twins. According to Write's report (1997), these

\footnotetext{
${ }^{10}$ In some (not infrequent) cases, it is possible to assess heritability as near to zero, even for traits clearly under strong genetic control. What is needed is a population in which the variability among individuals for a trait is not due to genetic differences, but environmental ones. For example, let us consider the fact that almost every individual has two arms, something strongly heritable. Now suppose that in a population every individual with one arm lost the second one during war. Under these circumstances, the variability among individuals would be due entirely to non-genetic factors. Thus, heritability of the two arms-phenotype would be zero.
} 
twins revealed very interesting resemblances when they met again as adults: not only similar IQs or personality traits, but also shared hobbies, political and religious preferences, similar partners, similar pets. Lastly, the magnitude of genetic factors seems to increase during development: the IQs of MZ twins correlate to each other more and more as they age. This seems to suggest that environmental factors become almost irrelevant during adulthood: "early in life, shared environmental factors are the dominant influence on IQ, but gradually genetic influence increases, with the effects of shared environment dropping to near zero" (Bouchard, 2004, p. 149). ${ }^{11}$

In these examples, we may easily recognize the idea that genes work prior to environment to canalize the organism's development: the core idea is that genes are capable of determining complex traits like intelligence. Often, this is assumed regardless of any reference to developmental mechanisms (see Tabery \& Griffiths, 2010) and without invoking any non-genetic influence as really relevant. While Griffiths identified a link between the innateness concept and folk essentialism, I explore the relationship between folk essentialism and the quantitative view. This view allows us to speak about genetic and environmental causal pathways as separate things-merely additive and quantifiable in percentage terms. Trivially, in order to think that inheritance plays a greater role than environment (e.g., 80 versus 20), one needs to think that the causal power of genes could be separable from that of the environment. This is closely reminiscent of folk essentialism: if, from a causal point of view, genes and environment can act separately, then the genome could be that underlying property capable of explaining individuals' phenotypic similarities.

One may notice that this assumption originates from a methodological requirement. Heritability research tends by definition to separate genes and environment. In fact, in order to analyze phenomena which are really complex in a natural context, a common strategy is to isolate variables. Nonetheless, this "carving perspective", separating genes and environment in development, originates from the folk essentialist ontological assumption made prior to heritability research. This was manifest in Galton's conceptual distinction between nature and nurture and has remained hidden through decades of research pursing different methodological purposes. Then, with behavioral genetics, the carving perspective became visible as an explicit ontological conception. Moving from the context of artificial selection with inbreeding plants to complex human traits as intelligence, a methodological artefact has been taken as a biological principle: the effect of genes and environment is merely additive and there is no relevant interaction between the two. Briefly, findings deriving from heritability research, based on an "artificial" distinction, have led several scholars to think that genes-environment interactions are negligible.

\footnotetext{
${ }^{11} \mathrm{My}$ aim is neither to defend nor to criticize these findings, but rather to describe those data which lead to the PSY-GEN approach.
} 
For a few decades, developmental biology has advanced convincing reasons to think that interactions between genes and environment play a chief role in development. ${ }^{12}$ In this respect, folk essentialism seems to arise more easily in a quantitative view than in an interactionist one: taking interactionism seriously, one cannot make a relevant distinction between the causal power of genes and of environment on phenotypes. Following such a line of reasoning, we need to abandon a quantitative view of intelligence in order to set aside this sort of essentialism with respect to intelligence. In the next section, I evaluate a kind theory of intelligence to analyze whether it would be more convincing than a quantitative one.

\section{Which Kind of Kind Theory?}

Not every type of kind theory is suitable for describing complex psychological phenomena like intelligence. According to Haslam, the traditional conception of natural kind applies rarely to psychology because it carries essentialist luggage. "Only some causal stories can produce categories that might qualify as natural kinds in the classic, essentialist sense. [The basis of a natural kind] is a single cause that is common to all category members and that directly gives rise to the kind's properties. In the psychiatric domain, for example, a discrete disorder whose clinical features ultimately derived from a specific neural or genetic dysfunction that was shared by all afflicted individuals would qualify as a natural kind in the sense intended here" (Haslam, 2014, p. 16). This essentialism for kinds can be found in molecular research.

\subsection{Essentialism via molecular research}

After many decades of studies committed to the estimation of heritability, behavioral genetics has adopted methods to analyze genetic resemblances among individuals by finding associations between phenotypes and genotypes. Presumably, if two people show the same features (like a mental disorder or an analogue IQ level), they must share some underlying genetic factor. ${ }^{13}$ The main target of these methods is to find specific genetic variants involved in the heritability of a given phenotypic trait-in our case, involved in the heritability of the $g$ factor (see Plomin et al., 2013, pp. 206-209, for some attempts). ${ }^{14}$

\footnotetext{
${ }^{12}$ See Hood et al. (Eds., 2010) on the contrast between the quantitative and the interactionist approach.

${ }^{13}$ This assumption would be true if heritability research could ensure that a highly heritable trait is influenced by genes. Unfortunately, molecular research assumed that heritability findings are reliable without further analyses (Joseph, 2004), even if several authors were unconvinced by them.

${ }^{14}$ I mainly focus on the candidate-gene approach, which is more related to traditional essentialism than genome-wide association studies (GWAS). Indeed, the candidate-gene approach seeks genes that are supposed to be individually important for a trait; conversely, GWAS scan the genome more systematically
} 
Such attempts might appear to contrast with a quantitative conception of intelligence, conceiving intelligence as being weakly influenced by many genes. In other words, since genes have small individual effects on intelligence, it seems hard to identify single alleles related to individual intellectual differences (see §1.2).

In order to shed light on this point-and to make sense of the geneticists' view-I suggest that we are dealing with a categorical reasoning applied atop a dimensional one. Accordingly, we should presume that a quantitative conception of intelligence admits of qualitative distinctions: on a quantitative view, no single gene plays a significant role for an individual's IQ level; however, it is possible to find specific genetic variants that are sufficient (and sometimes necessary) conditions linked to a particular IQ level. In order to clarify this point, let us consider two types of mental phenomena that are often taken to be equivalent: a general cognitive disability due to monogenic conditions and a general cognitive disability characterized by a threshold on a dimensional scale of values. Let us examine them in turn.

Some cognitive disabilities depend directly on inheritance. A classic example is phenylketonuria (PKU), a metabolic disorder in which the organism cannot properly metabolize phenylalanine. This amino acid is found in many types of food. So, in the absence of a specific diet, phenylalanine is stockpiled in PKU carriers' blood and brain, leading to altered neural development. Broadly speaking, one could say that the PKUrelated cognitive disability is due to a specific monogenic issue. ${ }^{15}$ In this case, we could perhaps legitimately frame PKU inside a categorical-essentialist perspective: every PKUrelated behavior (like low IQ) is linked to a specific biological feature. The category members share the same "causal story"-a particular genetic variant and a "normal" diet-which is a necessary and sufficient condition to develop the disease.

However, in the majority of cases, cognitive disability is the "negative" pole on a dimensional scale, the outcome of a complex individual story that involves several causal pathways (e.g., birth problems, nutritional deficiencies, head injuries, social or educational issues, see Plomin et al., 2013, p. 164). As a core difference, none of them would be individually necessary to obtain a low score on IQ tests. Thus, with respect to "dimensional disabilities", one cannot readily adopt a categorical-essentialist approach.

Behavioral genetics rarely makes this distinction explicit, treating dimensional variation in a categorical way, like monogenic conditions. ${ }^{16}$ The process by which molecular

and look for genes that are not supposed to be individually important. Hence, GWAS concern quantitative genetics and folk essentialism (see §2).

15 Actually, this is a simplification: what is genetically determined in PKU is the metabolic issue, not the cognitive disability.

${ }^{16}$ It has been often assumed that, if cognitive disabilities due to monogenic inheritance exist, then genes involved directly in IQ level should exist as well. This argument is, at best, questionable. There are conditions, like PKU, in which a single genetic variant plays a role in IQ level: specific alleles can be involved in dysfunctional developmental mechanisms which lead to an abrupt break in normal development-and, as a consequence, to low IQ. However, it does not follow that any IQ level (both 
genetics superimposes a categorical way of reasoning upon a dimensional one is now clear. Generally speaking, intelligence is conceived as a quantitative trait. However, the recognition that some genes could have, even if taken in isolation, an appreciable effect on intelligence-as in PKU-leads geneticists to think that some genes are more important than others in explaining $g^{2}$ s heritability. Molecular research-the candidate gene approach especially-tries to find them. ${ }^{17}$

Accordingly, one might think of the two types of cognitive disability differently in relation to essentialism. On the one hand, there is the dimensional phenomenon, that is related to quantitative genetics and heritability research, and is therefore tied to folk essentialism (\$2); on the other hand, there is the monogenic phenomenon. In this case we could identify traditional essentialism, insofar as carrying some genes would be a sufficient (and, in some cases, a necessary) condition to develop a cognitive disability. Thus, traditional essentialism is related to molecular research desiderata, according to which it might be ideally possible to reach a full knowledge of every gene involved in the development of intelligence.

\subsection{Avoiding traditional essentialism: Homeostatic Property Clusters}

Classical natural kinds theory is often found wanting with psychological phenomena (§3). Among several theoretical proposals devoted to replacing the classical view, one of the most accepted was introduced by Boyd. Boyd had two objectives. The first was to address Hacking's objection (1991), according to which natural kinds are minddependent. Hacking holds that our scientific taxonomies cannot trace the real structure of the world because boundaries depend on epistemic purposes. The second was to avoid essentialism about kinds and properties. Frequently, in the biological domain, one cannot identify necessary and sufficient conditions for a kind membership. Biological kinds "are more heterogeneous than elements in a periodic table. Unlike all atoms of gold, individual members of a species need not share all their properties" (Kendler et al., 2011, p. 1147). ${ }^{18}$

Boyd satisfied both of these objectives by postulating the existence of a homeostatic mechanism, a causal pathway that explains why properties are statistically clustered

within and outside the normal range of values) is always determined by the effect of specific genes.

${ }^{17}$ So far, molecular studies failed to deliver the expected results (see e.g., Chabris et al., 2012). Low replicability rates in molecular research have been taken as further evidence that genes' individual effect is small and hard to identify, leading to embrace GWAS (e.g., Plomin et al., 2009). I do not discuss theoretical issues concerning this methodological turn.

${ }^{18}$ Let us suppose a lack of any "deep" biological knowledge about species membership. For instance, we may refer to different tigers as belonging to the same kind by virtue of their surface properties. Nonetheless, we may also name 'tiger' a tiger which lacks many of those surface properties (e.g., a tiger without stripes, without claws and with just three legs). In fact, we tend to think that (at least some) surface properties are not relevant to define the nature of an individual and its species membership. 
together. Biological entities are often variable and unpredictable. The homeostatic mechanism grants enough flexibility to admit even huge variations among a kind's members. Furthermore, it grants stability to the kind, allowing us to ground prediction and inductive reasoning. Hence, good taxonomies might support successful scientific enterprises insofar as they are capable of tracing (some aspects of) the causal structure of the world, i.e., its homeostatic mechanisms.

So, concerning essentialism, Boyd's theory admits individual cases in which not every property is shown. Indeed, the essence of a kind does not involve properties. Rather, it involves, at most, the homeostatic mechanism. ${ }^{19}$ At the same time, Boyd tried to save the notion of natural kind as mind-independent-accounting for realism-by highlighting the difference between a mere set of properties and a natural kind. Such a difference consists in a non-artificial association of properties, based on the existence of a homeostatic mechanism. This causal link is very important: without it, properties would be unrelated with each other, forming a mere property set; but if causality is established, properties would form a cluster (Khalidi, 2014; Wilson et al., 2007). In this second case, a kind is capable of satisfying both ontological and epistemological requirements. ${ }^{20}$

As it is anti-essentialist yet realist HPC theory is a good candidate to characterize biological intelligence. Another important reason concerns multilevel analysis. Psychological phenomena show several properties, generally spread across different levels of organization-from behaviors to low-level mechanisms. Cognitive systems, in turn, are frequently described as hierarchically organized. This applies to psychometric intelligence as well: several models try to explain how different datasets, concerning different variables (e.g., neurobiological, cognitive and psychometric ones), could match with each other, maintaining the validity of the related scientific enterprises. In particular, these models seek for a plausible organization of different variables in relation with $g .{ }^{21}$

The $g$ factor may be understood as a causal link holding variables together. In order to make sense of this relationship among variables, the HPC theory is especially wellsuited. Indeed, this theory acknowledges a hierarchical relationship among a phenomenon (a property cluster) and its parts (e.g., properties and mechanisms), understood as causally related with each other.

Albeit attractive, Boyd's theoretical solution has been evaluated as explanatorily weak: indeed, the HPC theory is inadequate on its own in identifying those mechanisms which underlie a property cluster (Boyd, 1991; Craver, 2009; Wilson et al., 2007). This vagueness will be relevant later in the discussion.

\footnotetext{
${ }^{19}$ See Samuels (2007) and Khalidi (2015) for different positions on this point.

${ }^{20}$ This is also one of the reasons why HPC have taken root in philosophy of psychiatry: behaviors and clinical symptoms are conceived as observable properties occurring over more fundamental phenomena, i.e., underlying causal patterns.

${ }^{21}$ About hierarchical models of psychometric and cognitive variables, see Jensen (2002), Kray \& Frensch (2002) and Schneider \& Flanagan (2015).
} 
In the next section, I shall delineate how the HPC model of intelligence accounts for PSY-GEN's intuitions.

\section{A Kind Theory of Intelligence}

Consistently with what has been said above, an HPC theory of intelligence might have advantages over other views of natural kinds concerning traditional essentialism and multilevel analysis. Another potential merit is that it might allow the PSY-GEN view of intelligence to avoid folk-essentialism. I will investigate whether an HPC theory can avoid folk essentialism and whether it fits with some intuitions about cognition, concerning the relationship between IQ and cognitive processes.

Let us consider general cognitive disability as the "negative pole" on a value scale. Psychometrically, it is described as a variable conceptually comparable to normal IQ level-an IQ below 70. In this sense, disability is assessed quantitatively. From a genetic point of view, this is a phenotypic trait related to an additive genetic influence. In this respect, the only difference between a normal and a low IQ consists of carrying different alleles-and, of course, being subject to different environmental influences which, as we have seen, are generally less emphasized than genetic ones. According to the reasoning shown in $\S 1.2$, that is hard to analyze in terms of natural kinds.

Consider now two individuals who have the same low IQ score. Intuitively, even though they have an identical IQ, few would claim that their cognitive profiles would be identical or that their scores stemmed from similar causal mechanisms. If one tries to explain why an individual shows a low IQ, several explanations are possible. On a cognitive level, these individuals could have widely different abilities as manifest by different scores on different subtests-mathematical or logical or linguistic, etc. ${ }^{22} \mathrm{On}$ an etiological level, two similar IQs could depend on many factors combined in several ways-inheritance, trauma, education and social context. Briefly, two people affected by cognitive disability would presumably obtain a low IQ score for different reasons. Likewise, two people that are "successful" in IQ tests and placed in the same category might benefit from widely different causal mechanisms, related to different cognitive processes. If this is sound, then all the conditions are therefore in place for a kind theory of intelligence.

The HPC model I propose has five main characteristics. First, IQ depends on various

\footnotetext{
${ }^{22}$ The most widespread IQ test in the USA is the WAIS, introduced by Wechsler during the 1930s. It includes different subtests requiring different skills (mathematical, linguistic, logic, etc.). As explained in $\S 1$, test performances are statistically intercorrelated. However, the degree of these correlations varies largely (Stankov, 2002). This is consistent with the idea that different cognitive profiles may lead to similar IQ scores in different individuals. Moreover, "positive manifolds do not automatically reveal their meanings. For example, it is quite possible to obtain a positive manifold due to an overlap of task demands, rather than due to the influence of a general ability" (Kray \& Frensch, 2002, p. 186).
} 
cognitive processes, combined with one another in different ways in distinct individuals. Second, IQ is the measure of a behavior arising in an experimental context and not a cognitive phenomenon itself. Third, such cognitive processes are not individually necessary and jointly sufficient, for a given individual, to reach a particular IQ level. This saves us from traditional essentialism. Fourth, the HPC model maintains the core ideas of PSY-GEN, according to which intelligence is a general cognitive ability and $g$. plays a role. Fifth, the model is hierarchical in the manner proposed by psychometricians: domain-specific cognitive abilities (broad factors) are distinguished by $g$ (see $§ 3.2$ for details). Let us look at some details, shown graphically in Figure 1.

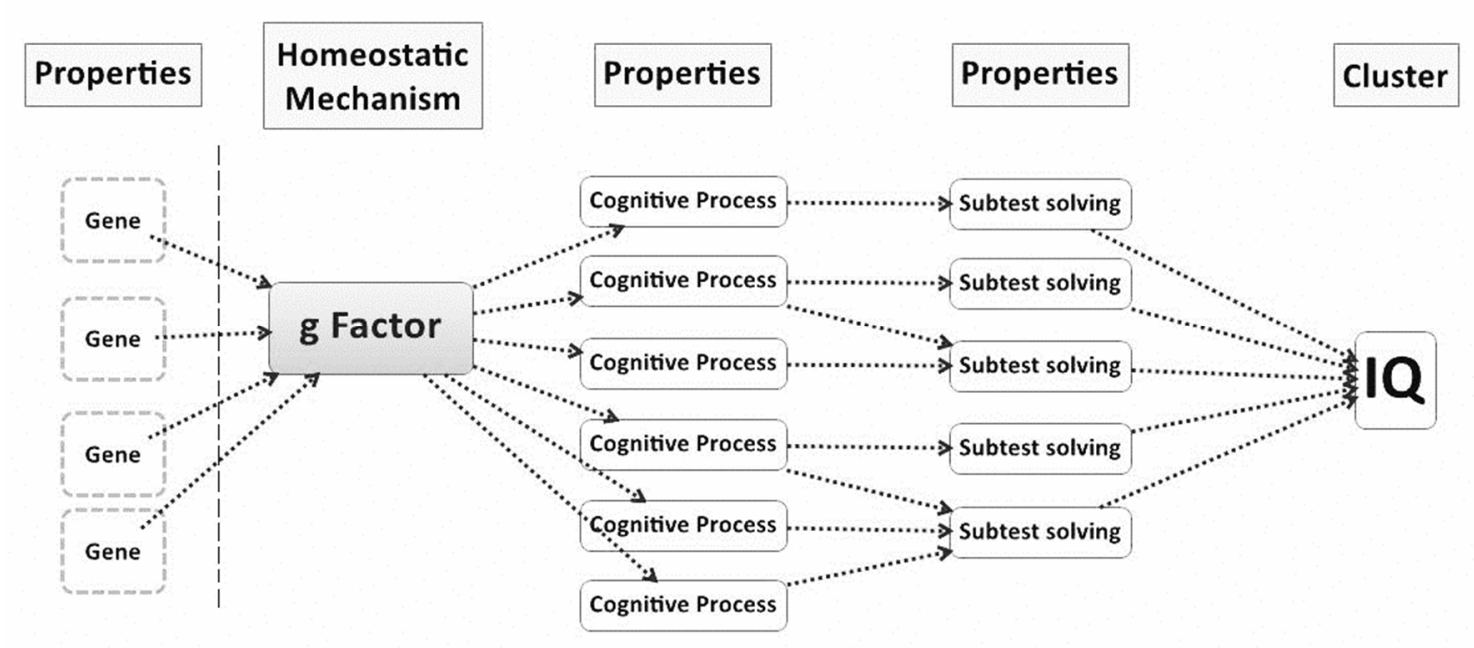

Figure 1

- Cluster. This is represented by IQ, which is useful in categorizing people. The IQ score is conceived as a behavioral output, and it represents the cluster. It may change or not among individuals, but every behavior related to IQ tests belongs to the same phenomenon. This is consistent with the intuition that intelligence is a unified thing.

- Properties: For a start, the property set includes performances related to subtests. I call them 'surface properties' because they are useful in categorizing individuals and in analyzing their cognitive profile. Other properties reside at deeper levels-e.g., those cognitive processes required to solve specific tasks. These processes presumably work differently in distinct individuals. Moving down a few steps, it is possible to suppose that biological factors, too, could represent the cluster's properties-e.g., some specific genes could be stably associated with a given IQ score. At once, one can suppose that the property set is joined by other biological correlates. ${ }^{23}$ Which properties are relevant in

\footnotetext{
${ }^{23}$ In Figure 1, there is a dotted line between deeper properties and the homeostatic mechanism because we still do not know what there is between the two-i.e., which biological correlates actually matter.
} 
categorizing people depends on the scientific target: surface properties would be more relevant in the psychometric context; cognitive processes would satisfy a psychological inquiry; biological properties are useful for biological analyses.

- Homeostatic mechanism: This is the $g$ factor, conceived as the source of the property cluster. For an HPC theory, it is necessary to individuate a homeostatic mechanism in order to be sure that a cluster is really a cluster and not a mere set of properties. This assumes that the properties have not been associated merely as a result of our predilections to lump certain properties together. The model assumes that IQ differences among individuals reflect differences in the functioning of $g$. So, it takes into account the flexibility-stability feature of intelligent behavior. The $g$ factor is a mechanism flexible enough to accept even considerable variations among individuals without appealing to different mechanisms for different categories (e.g., retardation, normality and genius). Nonetheless, the mechanism is stable enough to serve methodological purposes-e.g., generalization, prediction and identification of categories grouping together individuals similar to each other even if they do not exhibit the same surface properties. ${ }^{24}$ Summarily, $g$ allows us to explain why some properties are coinstantiated both in similar individuals and in widely different individuals, even analyzing the latter cases under the same phenomenon. In this sense, people with low and high IQ scores are dissimilar (at least with respect to surface and cognitive properties), but their differences do not mirror two (or more) distinct psychobiological mechanisms, but rather variations within a range of $g$ functioning.

What is the role of the term 'intelligence'? It is the kind itself, as distinct from the cluster. The cluster is a set of properties that are related for non-conventional reasons. Conversely, the kind represents intelligent behavior as a very broad phenomenon. Consistent with the geneticists' conception of intelligence as a phenotypic trait, all human beings participate in the kind. ${ }^{25}$

To summarize, if we assume that intelligence is a natural kind, and if we assume that $g$. exists, the HPC model sounds more promising than the quantitative view insofar as it accounts for the intuitions sketched above. Those intuitions remind us of a general trend in cognitive science, according to which cognition does not involve a general mental ability, but rather it consists of different domain-specific abilities (see Naglieri \& Das, 2002).

If the model sounds convincing, then intelligence is not a quantitative trait in itself; what

\footnotetext{
${ }^{24}$ This is a central point. We might assume a standpoint which is less metaphysically loaded than the one I assumed so far, by thinking of natural kinds from an epistemological perspective (for a discussion, see Magnus, 2012). In this respect, thinking of intelligence as a natural kind means thinking of it as an answer to methodological issues.

${ }^{25}$ One may try to extend the question to other species. It is worth mentioning that some authors (e.g., Jensen, 1980) accept that $g$ is a trait not solely limited to the human species.
} 
is quantitative is the IQ variation over populations. Ultimately, what is missing in the quantitative approach is a clear distinction between intelligence, IQ and $g$, which frequently collapse upon each other. IQ is a variable: one can measure it because it changes quantitatively within populations. This does not imply that intelligence is a unitary phenotypic trait describable by quantitative genetics. Furthermore, if the model is sound, then a traditional essentialist interpretation of intelligence seems untenable, insofar as one assumes that: 1) HPC theories describe psychological phenomena better than classical kind conceptions; and 2) HPC theories are capable of avoiding essentialism about properties-that is, properties are neither individually necessary nor jointly sufficient to ascribe an individual to a kind.

In the last section, I discuss the other side of the coin, challenging the temptation to lump several mental skills into a single comprehensive phenomenon. Thinking of intelligence as a set of many cognitive processes leads us to identify a set of widely heterogeneous behaviors without any empirical commitment to the psychobiological mechanisms involved. So, I shall argue that it is unnecessary to consider intelligence as a kind in the HPC or any sense.

\section{Is Intelligence Really a Kind?}

The HPC model seems suitable for accounting for properties according to an antiessentialist perspective, especially for subtest solving and cognitive processes. But is the model broad enough to say something about deeper-biological-properties and about the homeostatic mechanism? Is the genetic influence on $g$ quantitative or not? Is there any gene that is necessary and/or sufficient for the functioning of a particular $g$ ? These are empirical questions that we need to address.

Such questions likely remain unsolved because of the vagueness of Boyd's theory. As is noted in $\S 3.2$, the HPC theory does not itself identify those mechanisms that determine a property cluster. According to Boyd, the mechanism may or may not be underlying, it might derive from a single cause, it might involve several phenomena, and it might be internal or external. About psychopathology, Kendler and colleagues (2011, p. 1149) ask: "which of the diversity of possible causal processes should we emphasize when we construct our nosology?" It depends on the particular case under analysis. This is not solely a conceptual issue. Rather, it should be determined by empirical research: it depends on the particular case under examination and on which is the relevant scientific discipline.

So, it is an empirical question whether or not $g$ is a good candidate for the role of the homeostatic mechanism. Psychometrics alone cannot settle this. As is said in $\S 1$, it is generally assumed that $g$ arises as a stable phenomenon. Nonetheless, we need external validators: correlation matrices are not causally self-explanatory. If cognitive sciences are to play a role in this inquiry, then we should keep in consideration the disagreement 
about $g$ as a cognitive phenomenon (see $\S 1$ ). Indeed, if the opponents of $g$ are right, then the homeostatic mechanisms needed to ground an HPC theory of intelligence are lacking: thus, IQ would not be a cluster, but a mere set of properties. If this is the case, general intelligence would lose any epistemic advantage over other theoretical constructs for understanding of human cognition. It would, not be a rewarding posit to support epistemically successful science. Ontologically speaking, instead, the PSY-GEN approach to intelligence would be unable to carve nature at its joints. In both the epistemic and the ontological respects, intelligence would not be a natural kind.

Since the empirical findings are still under debate, we might advance alternative conceptual perspective that divides what seems prima facie a unified kind (intelligence) into different kinds (e.g., specific cognitive processes). Test solving seems to be due to many cognitive abilities and it is pointless to categorize them as a single thing (see Figure 2).

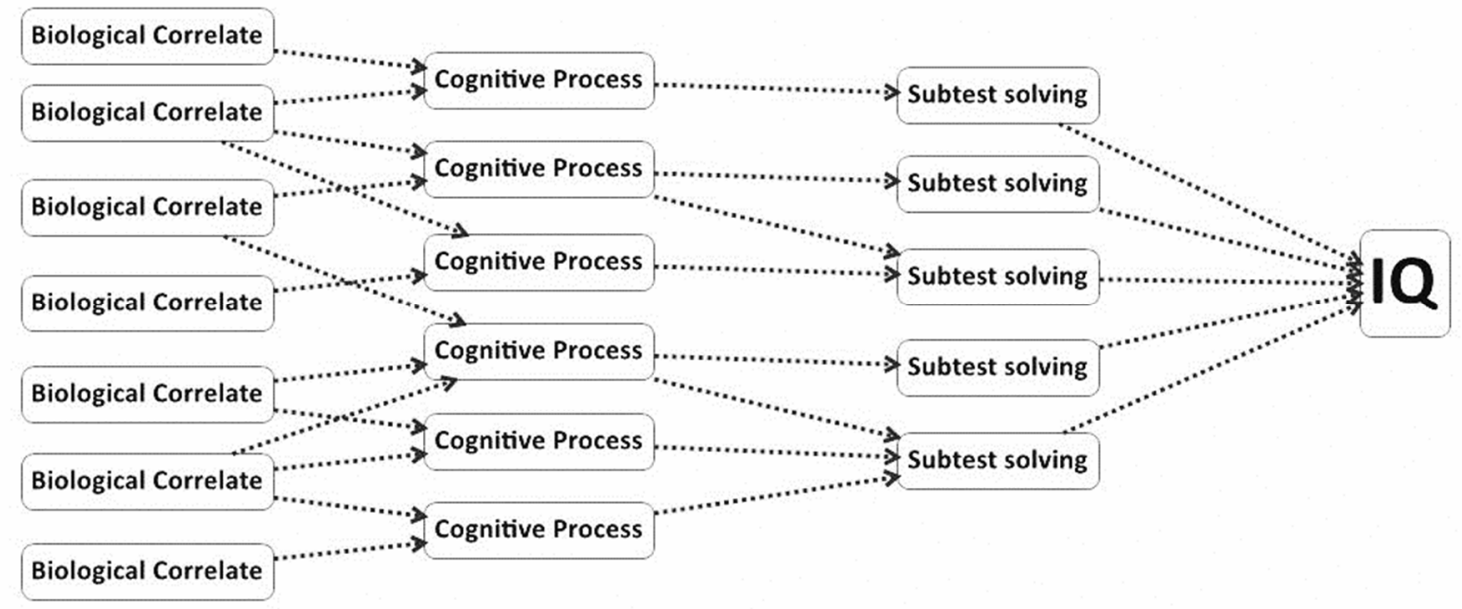

Figure 2

Accordingly, it seems empirically more productive to search for genes that act on specific cognitive components rather than genes that act on general intelligence. Some efforts have been made in this direction, but the overall trend is to maintain the hierarchical model of general intelligence, including $g$ as the main target of genetic influences (see Plomin et al., 2013, p. 217). By analogy, it would be as if we were looking for genes for the "escape velocity from predators" instead of genes for other phenotypic traits that matter somehow for speed. Of course, one can quantify comparative speeds (some individuals are faster than others), but it does not follow that speed is a quantitative trait. Van der Maas and colleagues (2014, p. 14) assume a similar position, noting that "if $g$ is not a causal source of the positive manifold, the search for a gene or brain area 'for $g^{2}$ will be fruitless. [...] The comparison with health is instructive. 
There are no specific genes 'for health', and health has no specific location in the body. Note that this line of reasoning does not apply to genetic and brain research on components of intelligence (for instance, working memory) as these components often do have a realistic reflective interpretation. Working memory capacity may very well be based on specific and independently identifiable brain processes, even if $g$ is not". The attempt to make sense of specific cognitive components as natural kinds is a matter of future research.

\section{Conclusion}

Focusing attention on empirical issues as assessed by neurobiological sciences, one can find convincing reasons to think that a quantitative view of intelligence is untenable. For instance, environmental and multilevel biological influences seem to be inseparable from one another. These data, which are less controversial than the ones related to the $g$ factor, point to an interactive model rather than to a purely additive one. In other words, the theory of biological intelligence seems to match in a slippery way both biological and cognitive data.

The HPC model provided an initially promising conceptual framework for analyzing ontological aspects within intelligence research. For instance, it has the merit of clarifying the relationship between IQ, cognitive processes, and $g$. However, the model leaves several questions about $g$ open, especially concerning causal aspects. An alternative framework that subtypes intelligence into cognitive processes satisfies intuitions concerning surface properties. But it does more than this: it rejects explanations based on $g$. Moreover, it seems capable of avoiding that sort of essentialism that follows easily from the quantitative perspective.

I conclude with a general observation. The recent endeavor to analyze psychological phenomena as natural kinds-and, then, as Boydian kinds-relies on the expectation that widespread concepts like intelligence must reflect some feature of the outside world. If we need a naturalistic theory of natural kinds, we should admit property clusters suitable for tracing the causal structure of the world. General intelligence does not seem to fulfill this requirement.

\section{References}

American Psychiatric Association (2013). Diagnostic and Statistical Manual of Mental Disorders (5th ed.). Arlington, VA: American Psychiatric Publishing.

Barrett, L. F. (2006). Are Emotions Natural Kinds? Perspective on Psychological Science, 1(1), 28-58.

Block, N. (1995). How heritability misleads about race. Cognition, 56, 99-128. 
Bouchard, T. J. (2004). Genetic Influence on Human Psychological Traits. A Survey. Current Directions in Psychological Science, 13(4), 148-151.

Bouchard, T. J., Lykken D. T., McGue, et al. (1990). Sources of Human Psychological Differences: The Minnesota Study of Twins Reared Apart. Science, 250(4978), 223228.

Boyd, R. (1991). Realism, Anti-Foundationalism and the Enthusiasm for Natural Kinds. Philosophical Studies, 61(1/2), 127-148.

Chabris, C. F., Hebert, B. M., Benjamin, D. J., et al. (2012). Most reported genetic association with general intelligence are probably false positive. Psychological Science, 20(10), 1-10.

Craver, C. (2009). Mechanisms and natural kinds. Philosophical Psychology, 22, 575594.

Franklin-Hall, L. R. (2015). Natural kinds as categorical bottlenecks. Philosophical Studies, 172, 925-948.

Gould, S. J. (1981). The Mismeasure of Men. New York: Norton \& Co.

Griffiths, P. E. (2002). What is Innateness? The Monist, 85(1), 70-85.

Hacking, I. (1991). A Tradition of Natural Kinds. Philosophical Studies, 61(1/2), 109126.

Haslam, N. (2014). Natural Kinds in Psychiatry: Conceptually Implausible, Empirically Questionable, and Stigmatizing. In H. Kincaid \& J. A. Sullivan (Eds.), Classifying Psychopathology. Mental kinds and natural kinds (pp. 11-28). Cambridge, US: MIT Press.

Hood, K. E., Halpern, C. T., Greenberg, G., \& Lerner, R. M. (Eds.). (2010). Handbook of Developmental Science, Behavior, and Genetics. Wiley-Blackwell.

Humphreys, L. G., \& Stark, S. (2002). General Intelligence: Measurement, Correlates, and Interpretations of the Cultural-Genetic Construct. In R. Sternberg \& E. Grigorenko (Eds.), The General Factor of Intelligence. How General is it? (pp. 87-116). Mahwah: Lawrence Erlbaum.

Jensen, A. R. (1969). How Much Can We Boost IQ and Scholastic Achievement? Harvard Educational Review, 39, 1-123.

Jensen, A. R. (1980). Bias in Mental Testing. New York: The Free Press.

Jensen, A. R. (2002). Psychometric g: Definition and Substantiation. In R. Sternberg \& E. Grigorenko (Eds.), The General Factor of Intelligence. How General is it? (pp. 39-54). Mahwah: Lawrence Erlbaum.

Joseph, J. (2004). The gene illusion: genetic research in psychiatry and psychology under the microscope. New York: Algora.

Kendler, K. S., Zachar, P., \& Craver, C. (2011). What kinds of things are psychiatric disorders? Psychological Medicine, 41, 1143-1150.

Khalidi, M. A. (2014). Naturalizing Kinds. In B. Bashour \& H. D. Muller (Eds.), Contemporary Philosophical Naturalism and Its Implications (pp. 115-135), New York: Routledge.

Khalidi, M. A. (2015). Innateness as a Natural Cognitive Kind. Philosophical Psychology, 29(3), 319-333. 
Kincaid, H. \& Sullivan, J. A. (Eds.). (2014). Classifying Psychopathology. Mental Kinds and Natural Kinds. Cambridge (US): MIT Press.

Kray, J., \& Frensch, P. (2002). A View From Cognitive Psychology: $g$-(G)host in the Correlation Matrix? In R. Sternberg \& E. Grigorenko (Eds.), The General Factor of Intelligence. How General is it? (pp. 183-220). Mahwah: Lawrence Erlbaum.

Magnus, P. D. (2012). Scientific Enquiry and Natural Kinds. From Planets to Mallards. Basingstoke: Palgrave Macmillan.

Mather, K. (1943). Polygenic Inheritance and Natural Selection. Biological Reviews, $18(1), 32-64$.

Naglieri, J., \& Das, J. (2002). Practical Implications of General Intelligence and PASS Cognitive Processes. In R. Sternberg \& E. Grigorenko (Eds.), The General Factor of Intelligence. How General is it? (pp. 55-84). Mahwah: Lawrence Erlbaum.

Plomin, R., DeFries, J. C., Knopik, V. S., \& Neiderhiser, J. N. (2013). Behavioral Genetics (6th ed.). New York: Worth Publishers.

Plomin, R., Haworth, C. M. A., \& Davis, O. S. P. (2009). Common disorders are quantitative traits. Nature Reviews Genetics, 10, 872-878.

Pretz, J. E., \& Sternberg, R. J. (2005). Unifying the Field: Cognition and Intelligence. In R. J. Sternberg \& J. E. Pretz (Eds.), Cognition and Intelligence (pp. 306-318). Cambridge: CUP.

Samuels, R. (2004). Innateness in Cognitive Science. Trends in Cognitive Sciences, 8(3), 136-141.

Samuels, R. (2007). Is innateness a confused notion? In P. Carruthers, S. Laurence, \& S. Stich (Eds.), The innate mind: Foundations and the future (Vol. 3, pp. 17-36). Oxford: OUP.

Schneider, W. J., \& Flanagan, D. P. (2015). The relationship between theories of intelligence and intelligence tests. In S. Goldstein, D. Princiotta \& J. A. Naglieri (Eds.), Handbook of Intelligence. Evolutionary Theory, Historical Perspective and Current Concepts (pp. 317-340). New York: Springer.

Stankov, L. (2002). g: A Diminutive General. In R. Sternberg \& E. Grigorenko (Eds.), The General Factor of Intelligence. How General is it? (pp. 19-38). Mahwah: Lawrence Erlbaum.

Spearman, C. (1904). 'General Intelligence', Objectively Determined and Measured. The American Journal of Psychology, 15(2), 201-292.

Tabery, J., \& Griffiths, P. E. (2010). Historical and Philosophical Perspectives on Behavioral Genetics and Developmental Science. In K. E. Hood, C. T. Halpern, G. Greenberg \& R. M. Lerner (Eds.), Handbook of Developmental Science, Behavior and Genetics (pp. 41-60). Wiley-Blackwell.

Van der Maas, H. L. J., Dolan, C. V., Grasman, R. P. P. P., et al. (2006). A dynamical model of general intelligence: the positive manifold of intelligence by mutualism. Psychological review, 113(4), 842-861.

Van der Maas, H. J. L., Kan, K. J., \& Borsboom, D. (2014). Intelligence Is What the Intelligence Test Measure. Seriously. Journal of Intelligence, 2, 12-15.

Visscher, P. M. (2008). Sizing up human height variation. Nature Genetics, 40(5), 489490. 
Wahlsten, D. (2002). The Theory of Biological Intelligence: History and a Critical Appraisal. In R. Sternberg \& E. Grigorenko (Eds.), The General Factor of Intelligence. How General is it? (pp. 245-277). Mahwah: Lawrence Erlbaum.

Wilson, R. A., Barker, M. J., \& Brigandt, I. (2007). When Traditional Essentialism Fails: Biological Natural Kinds. Philosophical Topics, 35(1/2), 189-215.

Wright, L. (1997). Twins: And What They Tell Us About Who We Are. New York: Wiley \& Sons.

Zachar, P. (2014) Beyond Natural Kinds: Toward a "Relevant" "Scientific" Taxonomy in Psychiatry. In H. Kincaid \& J. A. Sullivan (Eds.), Classifying Psychopathology. Mental kinds and natural kinds (pp. 74-104). Cambridge, US: MIT Press. 